\title{
Aplicación y análisis de un ciclo de mejora en el aula en la asignatura de "Ciencia y Tecnología del Medio Ambiente"
}

\section{Application and analysis of a classroom improvement cycle on the subject "Environmental Science and Technology"}

LUIS MATÍAS

ORCID: https://orcid.org/0000-0001-5603-5390

Universidad de Sevilla.

Departamento de Biología Vegetal y Ecología.

Facultad de Biología.

Imatias@us.es

DOI: http://dx.doi.org/10.12795/9788447231003.046

Pp.: 964-982 


\section{Contexto del ciclo de mejora en el aula}

El presente ciclo de mejora en el aula (CIMA) asociado al Curso General de Docencia Universitaria se ha implementado en la asignatura de "Ciencia y Tecnología del Medio Ambiente", materia obligatoria que se imparte durante el primer cuatrimestre en el segundo curso del Grado en Ingeniería Agricola de la Escuela Técnica Superior de Ingeniería Agronómica en la Universidad de Sevilla durante el curso académico 2020-2021. En esta asignatura se tratan los principios generales de la Ecología y sus implicaciones para las actividades agrícolas y ganaderas, asi como los métodos de identificación y corrección de los impactos de estas actividades sobre el medio. De este modo, a lo largo de esta asignatura se trata de aplicar el conocimiento científico sobre el funcionamiento de los ecosistemas a la futura actividad profesional de los alumnos, fomentando una actitud positiva frente a las prácticas de desarrollo sostenible y una buena praxis ambiental. En concreto, el CIMA se aplicó a las sesiones prácticas de la asignatura, centradas en la evaluación de impacto ambiental, que cubren dos horas semanales (miércoles de 12:30-14:30). El grupo general de teoría se divide en 3 sub-grupos de prácticas, con un máximo de 20 alumnos en cada uno de ellos. En este curso, la asistencia a prácticas es voluntaria, aunque ha superado el $85 \%$ durante el periodo que aquí se detalla. A pesar de que las clases teóricas de esta asignatura se han impartido online durante este curso, las sesiones prácticas se han mantenido de forma presencial durante el periodo de aplicación del ciclo de mejora. Para el diseño del presente CIMA se han seguido las indicaciones recibidas en el Curso General de Docencia Universitaria (Porlán, 2017), así como experiencias previas en esta asignatura (ver Cambrollé Silva, 2018 y Mancilla Leytón 2018).

Ciclos de Mejora en el Aula (2020). Experiencias de Innovación Docente de la US Esta obra se distribuye con la licencia Creative Commons 


\section{Diseño previo del CIMA}

\section{Contenidos}

Este ciclo de mejora coincide con el Bloque I de contenidos, que se centra en el marco legislativo sobre el que desarrollan los estudios de impacto ambiental. Básicamente, tratamos el contenido actitudinal del "desarrollo sostenible" como una práctica a implementar durante el futuro desempeño profesional del alumnado. Para ello, trabajamos una serie de contenidos estructurales como son los conceptos de "medio ambiente", en el que han de incluir al hombre como una especie más que interactúa con el medio y tener en cuenta los aspectos socioeconómicos relacionados con determinadas actividades como un elemento más del medio, el "impacto ambiental" como una consecuencia (positiva o negativa) de las acciones humanas en el entorno, y la "evaluación de impacto ambiental" como instrumento de evaluación de una determinada actividad y sobre el que se centrarán los siguientes bloques de contenidos. Además, se desarrollarán una serie de contenidos secundarios y de datos que definen el marco legislativo sobre el que se evalúa el impacto ambiental de determinadas actuaciones. El mapa de contenidos de este bloque, y su inter-relación se han resumido de forma esquematizada en la Figura 1.

Cada uno de los conceptos se introduce mediante una serie de preguntas activadoras -veánse cuadros grises en Figura 1- (García, Porlán y Navarro, 2017). El contenido de este bloque se desarrollará a partir de un ejemplo práctico de la planificación de una hipotética explotación avícola. Antes de la apertura, el promotor debe informarse de los trámites y permisos necesarios para su construcción. ¿A quién debe dirigirse? ¿Qué tipo de permisos se requieren? Además, la empresa está interesada en obtener una

Ciclos de Mejora en el Aula (2020). Experiencias de Innovación Docente de la US Esta obra se distribuye con la licencia Creative Commons 
etiqueta "ECO" en sus productos a fin de dirigir sus ventas a un tipo de clientes con una sensibilidad ambiental moderada o alta. ¿Podemos considerar esa actividad como un tipo de desarrollo "sostenible"?

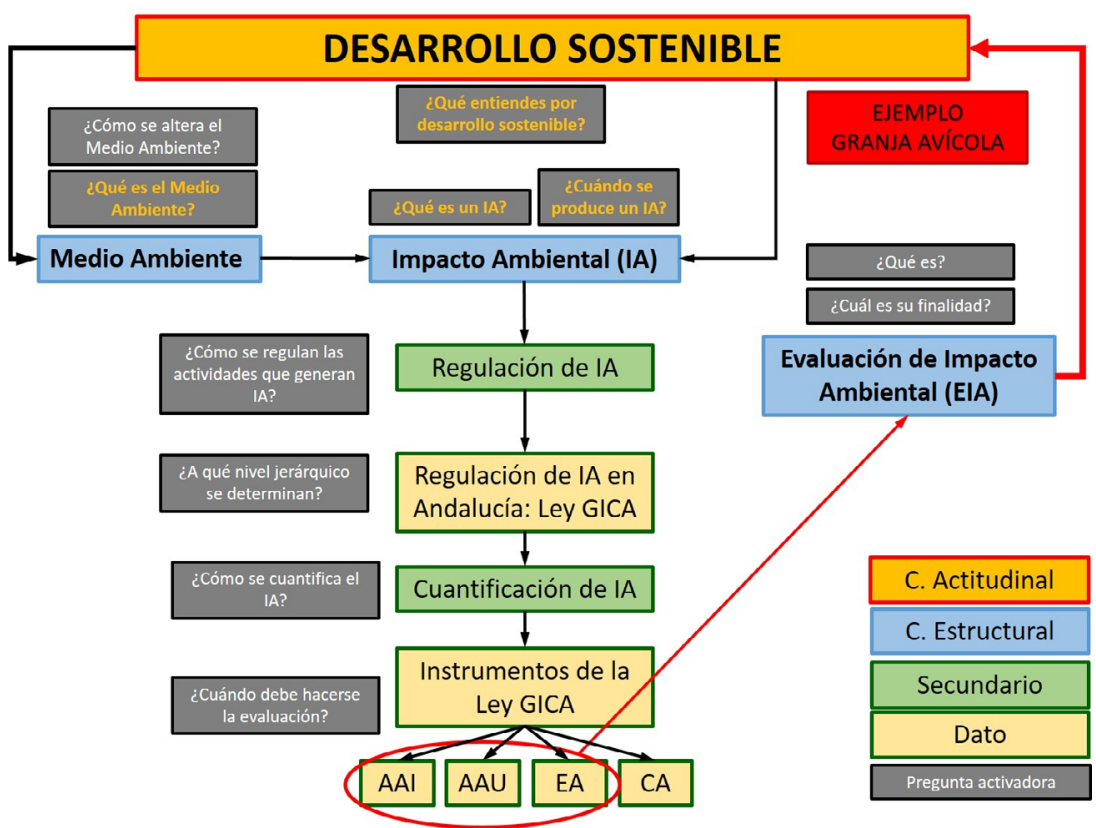

Figura 1. Mapa de contenidos a desarrollar durante el segundo ciclo de mejora en el aula. Además de los contenidos, se señalan las preguntas activadoras (claves en naranja y secundarias en blanco) en los cuadros de color gris.

\section{Modelo metodológico}

El modelo metodológico a emplear en este segundo CIMA (Figura 2) se basa en el trabajo y desarrollo de contenidos a partir de un caso práctico (CP) al que los alumnos podrían enfrentarse en su ámbito profesional una vez finalizada su formación. Una vez presentado este supuesto, los alumnos debatirán sus ideas iniciales $\left(I A_{i}\right)$ para resolver el problema planteado. Mediante el trabajo dirigido a través

Ciclos de Mejora en el Aula (2020). Experiencias de Innovación Docente de la US Esta obra se distribuye con la licencia Creative Commons 
de las preguntas activadoras presentadas en el cuadro de contenidos, los alumnos irán debatiendo los contenidos estructurales y secundarios de este bloque, seguidos de una pequeña recapitulación final por parte del profesor. Según las recomendaciones de Finkel (2008), estas actividades de contraste $\left(\mathrm{AC}_{1-\mathrm{n}}\right)$ deberían idealmente trabajarse en forma de pequeños grupos de 3-4 alumnos para hacer una puesta en común posterior entre todos ellos. Sin embargo, dada la situación sanitaria por la que atravesamos durante el curso académico de implementación del CIMA y, a fin de mantener la distancia de seguridad recomendada entre alumnos y dado que el número de alumnos no es muy elevado (20), creo que un debate grupal será la mejor opción para el desarrollo de las sucesivas actividades de contraste. Una vez cubiertos todos los contenidos, se les pidió a los alumnos que respondiesen de nuevo al cuestionario que se les pasó en la primera clase $\left(I A_{f}\right)$, lo que permitirá evaluar la adquisición de conocimientos. Por último, se finalizó la sesión con una síntesis (S) de los conceptos más relevantes de los incluidos en este bloque por parte del profesor.

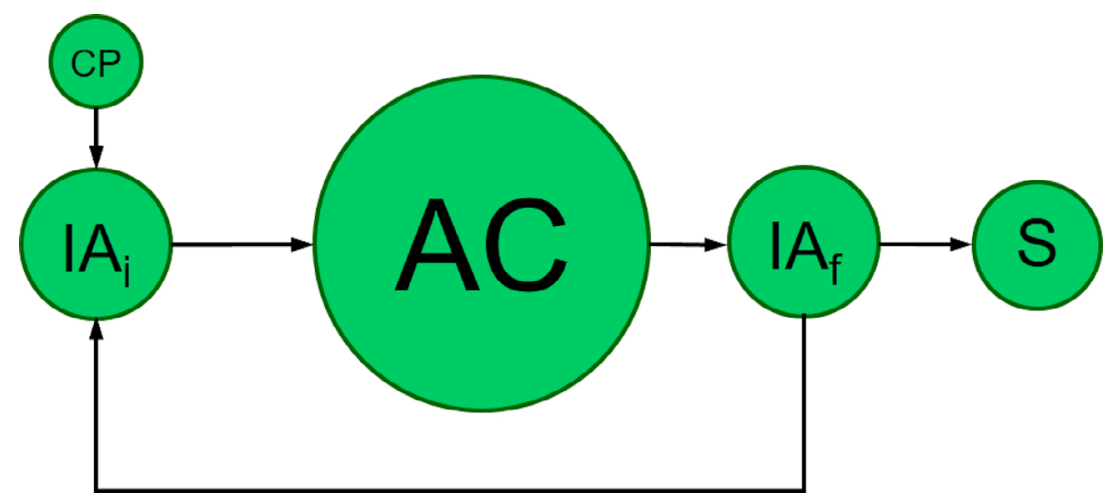

Figura 2. Modelo metodológico a desarrollar durante el segundo CIMA. CP: Caso práctico; $I A_{i}$ : ideas iniciales de los alumnos; AC: actividad de contraste; $\mid A_{\mathrm{f}}$ : ideas finales de los alumnos; S: síntesis.

Ciclos de Mejora en el Aula (2020). Experiencias de Innovación Docente de la US Esta obra se distribuye con la licencia Creative Commons 


\section{Secuencia de actividades}

El CIMA se implementó en tres sesiones de 2 horas de duración, que inicialmente se plantearon según las actividades detalladas en la siguiente tabla:

Tabla 1. Secuencia de actividades del ciclo de mejora en el aula

\begin{tabular}{|c|c|c|}
\hline \multicolumn{3}{|c|}{ SESIÓN $1(21 / 10 / 2020)$} \\
\hline Actividad & Descripción & $\begin{array}{l}\text { Tiempo } \\
\text { (minutos) }\end{array}$ \\
\hline Presentación & $\begin{array}{l}\text { Esta es la primera sesión de clase con los } \\
\text { alumnos, por lo que comenzaremos con } \\
\text { una presentación del profesor y de los } \\
\text { temas a desarrollar durante las sesiones } \\
\text { prácticas. }\end{array}$ & 15 \\
\hline $\begin{array}{l}\text { Normas } \\
\text { evaluación }\end{array}$ & $\begin{array}{l}\text { Es importante aclarar las normas de } \\
\text { evaluación, que se han consensuado con } \\
\text { los profesores de los distintos grupos. }\end{array}$ & 15 \\
\hline $\begin{array}{l}\text { Presentación } \\
\text { caso práctico }\end{array}$ & $\begin{array}{l}\text { Presento un supuesto práctico de una } \\
\text { persona que planea abrir una explotación } \\
\text { avícola y se plantea una serie de } \\
\text { cuestiones prácticas. }\end{array}$ & 5 \\
\hline Cuestionario & $\begin{array}{l}\text { Realizo el cuestionario de ideas previas de } \\
\text { los alumnos. }\end{array}$ & 10 \\
\hline $\begin{array}{l}\text { Actividad de } \\
\text { contraste } 1\end{array}$ & $\begin{array}{l}\text { Desarrollo de los conceptos de "Desarrollo } \\
\text { sostenible" y de "Medio ambiente" } \\
\text { mediante preguntas activadoras y debates } \\
\text { grupales. }\end{array}$ & 35 \\
\hline Sintesis $\mathrm{AC}_{1}$ & Conceptualización de las principales ideas. & 5 \\
\hline $\begin{array}{l}\text { Actividad de } \\
\text { contraste } 2\end{array}$ & $\begin{array}{l}\text { Desarrollo del concepto de “Impacto } \\
\text { ambiental" mediante preguntas } \\
\text { activadoras y debates grupales. }\end{array}$ & 30 \\
\hline Sintesis $A C_{2}$ & Conceptualización de las principales ideas. & 5 \\
\hline
\end{tabular}

SESIÓN $2(28 / 10 / 2020)$

\begin{tabular}{|l|l|l|}
\hline Actividad & Descripción & $\begin{array}{l}\text { Tiempo } \\
\text { (minutos) }\end{array}$ \\
\hline
\end{tabular}

Ciclos de Mejora en el Aula (2020). Experiencias de Innovación Docente de la US Esta obra se distribuye con la licencia Creative Commons 


\begin{tabular}{|l|l|l|}
\hline Recapitulación & $\begin{array}{l}\text { Repaso de la sesión anterior para retomar } \\
\text { el caso práctico y las preguntas a las que } \\
\text { ya habíamos respondido. }\end{array}$ & 10 \\
\hline $\begin{array}{l}\text { Actividad de } \\
\text { contraste 3 }\end{array}$ & $\begin{array}{l}\text { Desarrollo de contenidos sobre regulación } \\
\text { ambiental a distintos niveles jerárquicos } \\
\text { (desde europeo hasta local) mediante } \\
\text { preguntas activadoras y debates grupales. }\end{array}$ & 35 \\
\hline Síntesis AC & Conceptualización de las principales ideas. & 5 \\
\hline $\begin{array}{l}\text { Actividad de } \\
\text { contraste 4 }\end{array}$ & Regulación ambiental en Andalucía & 30 \\
\hline $\begin{array}{l}\text { Actividad de } \\
\text { contraste 5 }\end{array}$ & $\begin{array}{l}\text { Instrumentos de la Ley GICA. Aportación } \\
\text { teórica de contenidos tipo datos. Trabajo } \\
\text { en grupos (3 alumnos). Localización de } \\
\text { la autorización necesaria de distintas } \\
\text { actividades en el Anexo III de la Ley GICA. }\end{array}$ & 35 \\
\hline Síntesis AC & Conceptualización de las principales ideas. & 5 \\
\hline
\end{tabular}

\begin{tabular}{|l|l|l|}
\hline SESIÓN 3 (04/11/2020) & $\begin{array}{l}\text { Tiempo } \\
\text { (minutos) }\end{array}$ \\
\hline Actividad & Descripción & 10 \\
\hline Recapitulación & $\begin{array}{l}\text { Repaso de la sesión anterior para retomar } \\
\text { el caso práctico y las preguntas a las que } \\
\text { ya habíamos respondido. }\end{array}$ & 25 \\
\hline $\begin{array}{l}\text { Actividad de } \\
\text { contraste 6 }\end{array}$ & $\begin{array}{l}\text { Desarrollo del concepto de “Estudio } \\
\text { de Evaluación de Impacto ambiental" } \\
\text { mediante preguntas activadoras y debates } \\
\text { grupales. }\end{array}$ & 35 \\
\hline Síntesis AC 6 & Conceptualización de las principales ideas. & 5 \\
\hline Teoría & $\begin{array}{l}\text { Presentación de las distintas partes que } \\
\text { debe incluir un estudio de evaluación de } \\
\text { impacto ambiental. }\end{array}$ & 10 \\
\hline $\begin{array}{l}\text { Actividad de } \\
\text { contraste 7 }\end{array}$ & $\begin{array}{l}\text { Aplicación de todos los contenidos del } \\
\text { bloque para la resolución de las preguntas } \\
\text { planteadas en el caso práctico inicial. }\end{array}$ & 20 \\
\hline Síntesis AC & Conceptualización de las principales ideas. & 5 \\
\hline $\begin{array}{l}\text { Cuestionario } \\
\text { final }\end{array}$ & $\begin{array}{l}\text { Realizo el cuestionario de ideas finales de } \\
\text { los alumnos. }\end{array}$ & 10 \\
\hline Síntesis final & $\begin{array}{l}\text { Recapitulación final de contenidos por } \\
\text { parte del profesor. }\end{array}$ & 10 \\
\hline & & \\
\hline
\end{tabular}

Ciclos de Mejora en el Aula (2020). Experiencias de Innovación Docente de la US Esta obra se distribuye con la licencia Creative Commons 


\section{Cuestionario de diagnóstico}

Para poder evaluar la adquisición de conceptos clave por parte de los alumnos, realizamos un breve cuestionario de conceptos unidos al ejemplo de trabajo presentado al inicio de la sesión. Se les pidió además a los alumnos que rellenasen ese mismo cuestionario una vez finalizado el bloque de contenidos.

El cuestionario de ideas iniciales (o finales) de los alumnos se completó online a través de la plataforma Google Forms. Dicho cuestionario contiene las siguientes preguntas clave:

1. ¿Qué entiendes por "desarrollo sostenible"?

2. ¿Debe una explotación avícola ser sostenible?

3. Usando tus propias palabras, ¿qué entiendes por medio ambiente?

4. ¿Qué entiendes por "impacto ambiental"?

5. ¿Qué impactos puede producir la explotación avícola del ejemplo?

\section{Aplicación del CIMA}

\section{Resumen de las sesiones}

El inicio de la aplicación de este segundo CIMA coincidió con mi primer contacto con la clase, además de ser mi primer contacto presencial con los alumnos después de la pandemia, lo que rompía un poco con la experiencia de años anteriores y hacía un poco más imprevisible determinar de antemano lo que me iba a encontrar en clase. Es un grupo pequeño, de 20 alumnos, y lo primero que hago es pasar lista porque desde el centro me piden que deje constancia de la posición de cada alumno en el aula. Eso

Ciclos de Mejora en el Aula (2020). Experiencias de Innovación Docente de la US Esta obra se distribuye con la licencia Creative Commons 
también me ayuda a poner caras a los nombres, algo que este año va a ser mucho más difícil con el uso de las mascarillas. Al ser mi primera clase con ellos, lo primero que hago es presentar el programa de esta parte de la asignatura y los criterios de evaluación consensuados entre los profesores de los distintos grupos. Durante esta primera parte soy yo el que habla principalmente, con algunas pequeñas intervenciones puntuales de algún alumno para aclarar alguna cuestión o resolver dudas respecto a su situación personal. Esta primera parte es muy similar al desarrollo en cursos anteriores, salvo por las mascarillas y las distancias entre mesas.

Una vez que los aspectos formales han quedado claros, presento el ejemplo práctico sobre el que girarán los contenidos de este bloque, donde dejo en el aire una serie de cuestiones que iremos resolviendo durante esta y las siguientes sesiones. A continuación, les dejo el enlace al cuestionario de ideas previas en Google Forms, con 5 cuestiones. Deben contestarlo a través del ordenador o del móvil, lo que lleva un poco más de los 10 minutos inicialmente planeados. Una vez que todos han contestado, entramos por fin en el desarrollo de contenidos, planteando los conceptos de "desarrollo sostenible" y de "medio ambiente". Lo hago a través de las preguntas activadoras incluidas en el CIMA, tratando que los alumnos vayan desarrollando los conceptos por sí mismos. Al principio les cuesta mucho romper el hielo y la participación es muy baja, por lo que tengo que animarlos diciendo que no hay respuestas incorrectas $\mathrm{y}$, a veces, preguntando directamente a algún alumno en concreto para que responda. Poco a poco, van surgiendo ideas, con lo que los dos conceptos quedan bien definidos. Pasamos a continuación a tratar el concepto de "impacto ambiental", también a través de debates comunes. En este caso parece que ya se han animado un poco más a hablar en clase, aunque hay un par de alumnos que tienden a participar mucho más que el resto, por lo que trato de hacer que todos intervengan al menos una vez. Voy con cierto retraso respecto al programa, por lo que

Ciclos de Mejora en el Aula (2020). Experiencias de Innovación Docente de la US Esta obra se distribuye con la licencia Creative Commons 
tengo que dirigir un poco el debate para que no se disperse, pero terminamos todos los contenidos planificados para la sesión.

En la segunda clase, comenzamos de forma similar a la primera, recogiendo las posiciones de los alumnos y haciendo un repaso de los tres conceptos principales vistos en la sesión anterior. Compruebo que me dan una idea bastante acertada de cada uno de los tres conceptos con bastante rapidez, por lo que creo que los asimilaron bastante bien. El contenido de esta segunda sesión es bastante más árido y se presta menos al debate, ya que se centra sobre la legislación ambiental. Además, es una parte más alejada de mi propia formación, por lo que me siento menos cómodo. De todas formas, comenzamos debatiendo sobre temas más generales de organización de las leyes, desde nivel europeo hasta local, para poder centrarnos en el nivel sobre el que trabajaremos. En esta sesión me cuesta mucho más que participen los alumnos, no sé si por el tema en sí o por que no he sabido plantear las preguntas correctas. Creo que mi incomodidad con el tema se refleja en el desarrollo de la clase y la participación de los alumnos. Dado que los contenidos de esta sesión son principalmente de tipo "dato" y a la menor participación de los alumnos, tiendo a llenar yo más el tiempo. Al final de la sesión, les pido a los alumnos que preparen un ejercicio voluntario para trabajar durante la semana que deberán entregarme en el que examinen en detalle el Anexo III de la Ley de Gestión de Calidad Ambiental de Andalucía y respondan a unas preguntas relacionadas con el ejemplo central del bloque presentado en la sesión anterior. Ese ejercicio contará con un punto sobre la nota de este bloque.

Al inicio de la tercera sesión, en la que se cierra el bloque de contenidos, comienzo con un repaso general de los conceptos generales presentados en las dos sesiones 
anteriores. Hago también una breve presentación de las impresiones que he sacado del ejercicio que me han ido entregando a lo largo de la semana. Esta actividad de trabajo en casa no la tenía contemplada dentro del CIMA, pero creo que ha sido muy positiva dada la participación y la respuesta recibida. El contenido de esta sesión se centra sobre las distintas autorizaciones ambientales y la herramienta del estudio de impacto ambiental. Poco a poco, a través de las preguntas activadoras y con una mayor participación de los alumnos en esta sesión, vamos viendo qué actividades requieren cada una de las distintas autorizaciones ambientales que contempla la ley autonómica y en qué se diferencian entre sí. A continuación, analizamos las partes de los estudios de impacto ambiental, que será la parte central de los dos siguientes bloques de contenidos. Compruebo la hora y veo que voy un poco por encima de lo previsto, pero nada demasiado alarmante. A continuación, retomamos el ejemplo práctico presentado en la primera sesión y entre todos se van respondiendo a las distintas cuestiones planteadas. Algunas, como la del tipo de autorización que requiere la granja propuesta, son muy directas y presentan poco debate porque se han ido respondiendo con los contenidos. Sin embargo, otras como la de si una granja de este tipo debe ser sostenible, generan un mayor debate sobre el concepto de sostenibilidad y su aplicabilidad en su actividad profesional. El debate es bastante fluido, y lo corto 10 minutos antes del final de clase para pedirles a los alumnos que respondan de nuevo al cuestionario de ideas finales.

\section{Evaluación del aprendizaje}

A partir de las preguntas planteadas al inicio y al final del ciclo de mejora, se realiza un análisis comparativo siguiendo la metodología detallada en Rivero y Porlán (2017). A continuación se detallan las escaleras de aprendizaje generadas a partir de las respuestas de los estudiantes, categorizadas en niveles crecientes de complejidad (A-D). En

Ciclos de Mejora en el Aula (2020). Experiencias de Innovación Docente de la US Esta obra se distribuye con la licencia Creative Commons 
dichas escaleras, podemos comprobar, además, la evolución del conjunto de alumnos de la clase desde las ideas iniciales (porcentajes en rojo) a las finales (porcentajes en negrita). En ellas podemos ver los distintos modelos mentales (grados de verde) y los obstáculos que pueden encontrar los alumnos para avanzar de escalón (Figuras 3 a 7). De los 20 alumnos del grupo, 16 respondieron al cuestionario inicial, mientras que sólo 11 lo hicieron al cuestionario final.
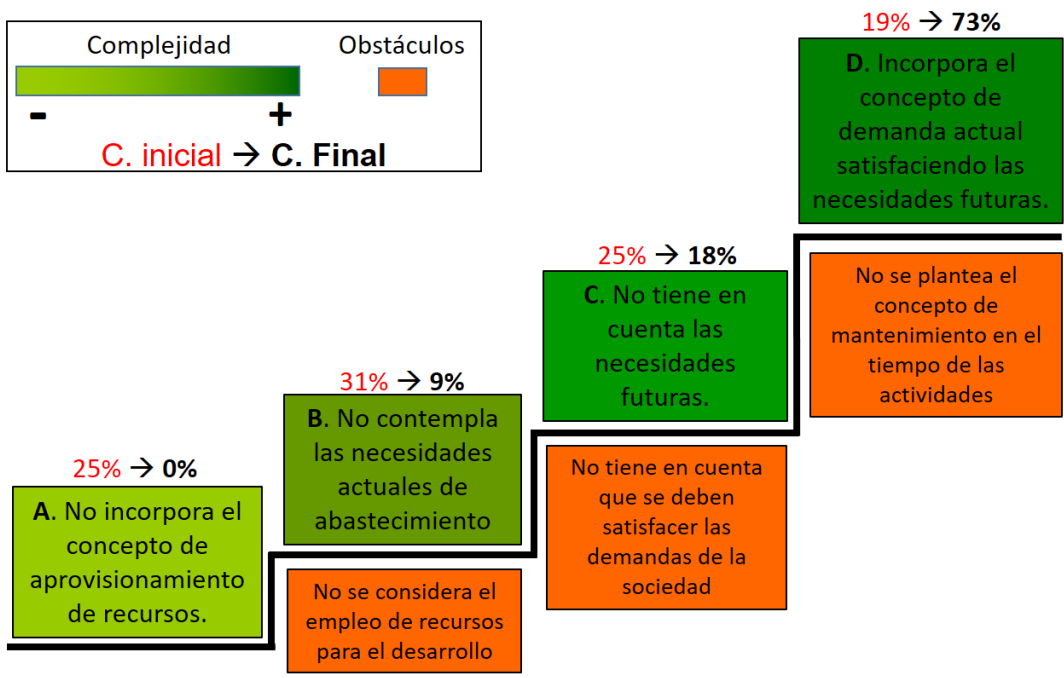

Figura 3. Resultados de la pregunta 1. ¿Qué entiendes por "desarrollo sostenible"?

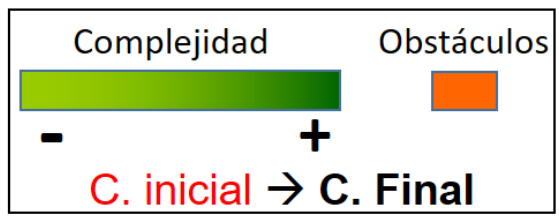

\section{$12 \% \rightarrow 55 \%$}

C. Usa criterios de

sostenibilidad

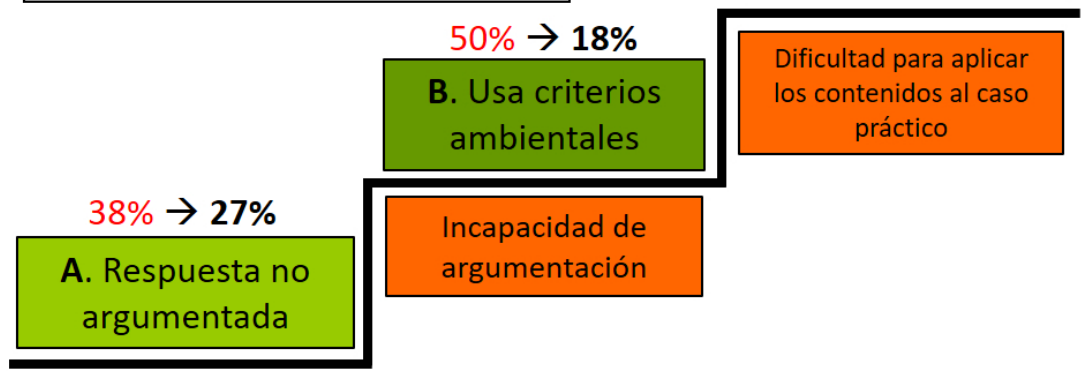

Figura 4. Resultados de la pregunta 2. ¿Debe una explotación avícola ser sostenible? Ciclos de Mejora en el Aula (2020). Experiencias de Innovación Docente de la US Reconocimiento-NoComercial-SinObraDerivada Internacional (CC BY-NC-ND 4.0.) 


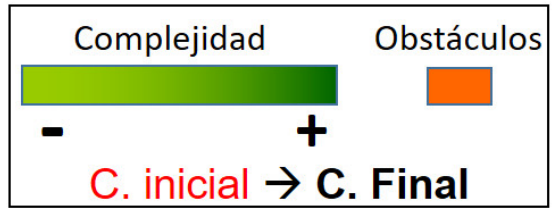

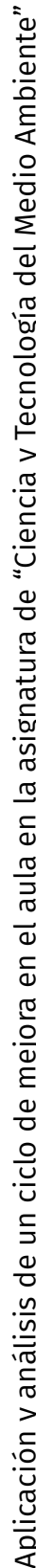

cambiental"?
$50 \% \rightarrow \mathbf{2 7} \%$

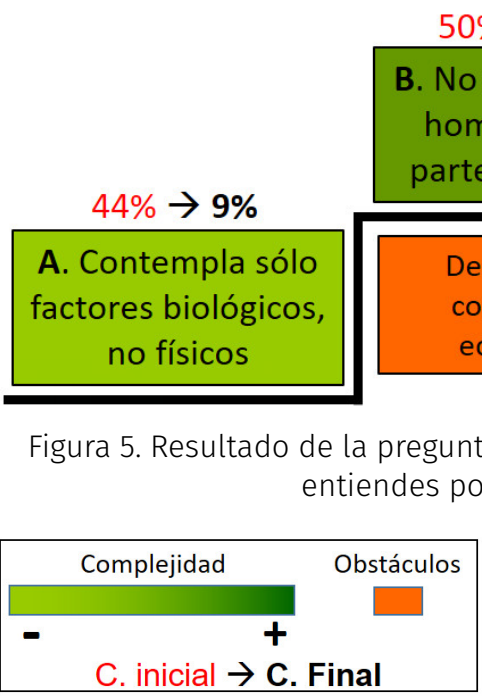

$31 \% \rightarrow 9 \%$

$19 \% \rightarrow 0 \%$

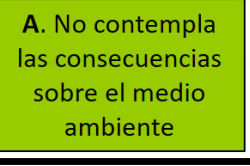

Figura 6. Resultado de la pregunta 4. ¿Qué entiendes por “impacto
ambiental”?

Figura 6. Resultado de la pregunta 4. ¿Qué entiendes por "impacto
ambiental"?
Desconoce el concepto de ecosistema
B. No contempla la causa humana como origen

Falta desarrollar el concepto de impacto
$44 \% \rightarrow 18 \%$

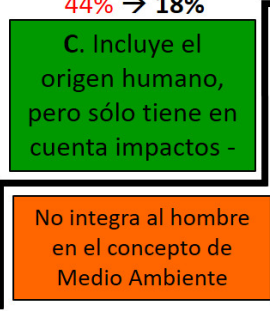

No se contempla e componente social

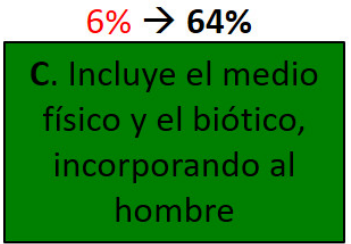

Falta incorporar

hombre como parte esencial del medio ambiente.
$6 \% \rightarrow 73 \%$

D. Tiene en cuenta

el origen humano y

los impactos $+y$

Ciclos de Mejora en el Aula (2020). Experiencias de Innovación Docente de la US 

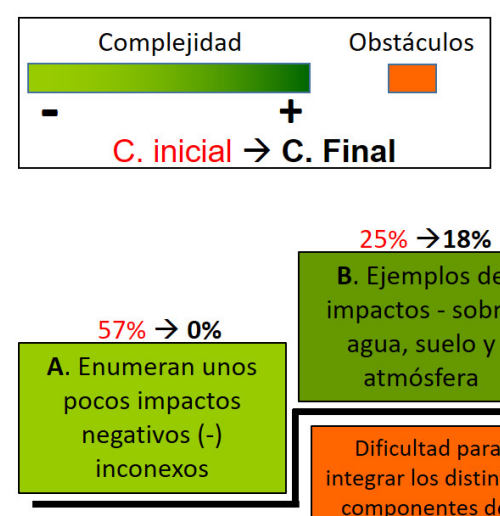

Figura 7. Resultado de la pregunta 5. ¿Qué impactos puede producir la explotación avícola del ejemplo?

A partir de estos resultados podemos comprobar cómo han evolucionado los modelos mentales de los estudiantes durante la implementación de este ciclo de mejora. Las respuestas a cada pregunta se han categorizado en 3 o 4 niveles de complejidad, y se han identificado además los principales obstáculos que dificultan el aumento de nivel de las respuestas. Como vemos en los resultados del cuestionario inicial (datos representados en color rojo sobre los cuadros verdes), la mayoría de los estudiantes se sitúan en los dos peldaños inferiores antes del inicio de las sesiones, lo que implica un grado de conocimiento medio-bajo de los temas a desarrollar en este bloque de contenidos. Tras el desarrollo de las diferentes actividades de contraste empleadas en el CIMA, comprobamos que la mayoría de los alumnos han alcanzado el mayor nivel de complejidad en todas las preguntas (porcentajes en negrita sobre los recuadros verdes). En 3 de las 5 preguntas no encontramos ninguna respuesta en el nivel más bajo en el cuestionario final, y en una de ellas sólo 1 alumno permanece en dicho escalón. Sin embargo, cabe destacar la pregunta número 2 por el significativo número de estudiantes 
en el nivel de complejidad más bajo (3 de 11 respuestas, $27 \%$ ) debido a que sólo han contestado con un escueto "sí" sin argumentar su respuesta ni en el cuestionario inicial ni en el final. Las preguntas 2 y 5 , ambas relacionadas con la aplicación de los contenidos a un caso práctico, son las que han tenido un menor éxito, con un porcentaje elevado de alumnos situado en niveles intermedios. Tanto en temas posteriores como en los siguientes cursos, se ha de hacer más hincapié en la aplicación directa de los conceptos tratados en clase a situaciones profesionales.

\section{Evaluación del CIMA}

\section{Cuestiones a mantener y cambios a introducir en el futuro}

Tras la implantación de este ciclo de mejora en el aula, creo que lo que mejor ha funcionado ha sido el mantener un ejemplo práctico que dé cabida a todos (o al menos a la mayoría) de los contenidos y los trabajos en casa. El ejemplo práctico ha permitido poner en contexto los contenidos teóricos, de forma que los alumnos puedan comprobar "en tiempo real" la aplicabilidad de la información con la que trabajamos en clase. El trabajo individual en casa hizo que se trabajase en mayor profundidad un aspecto muy concreto pero importante de los contenidos, de forma que los alumnos han adquirido mayor familiaridad con él. En esta ocasión se les pidió a los alumnos un trabajo de forma individual, pero según lo expuesto por Finkel (2008), creo que en futuras ocasiones sería más beneficioso que se trabaje por pequeños grupos de forma que se contemplen diversos puntos de vista durante el desarrollo de la tarea. El desarrollo de los conceptos principales mediante preguntas activadoras y debate general de los alumnos ha sido muy fructífero en la mayoría de

Ciclos de Mejora en el Aula (2020). Experiencias de Innovación Docente de la US Esta obra se distribuye con la licencia Creative Commons 
las ocasiones. Sin embargo, durante la segunda sesión no funcionó del todo bien. No sé si pudo ser por el tema en sí que daba poca opción de debate o por mi propia actitud frente a esos contenidos, pero de cara al próximo curso esta sesión hay que abordarla desde otra perspectiva para tratar de hacerla más atrayente. Probablemente un trabajo en grupos pequeños en el que cada grupo trabaje una parte específica de forma autónoma y después se haga una puesta en común sea una buena opción para abordar este contenido.

\section{Aspectos a incorporar en la práctica docente habitual}

De cara a mi práctica habitual, creo que el uso de un ejemplo común e integrador para todo un bloque de contenidos ha sido algo muy positivo. Si además ese mismo ejemplo o caso práctico pudiese emplearse en más de un bloque de contenidos para trabajarse desde distintas perspectivas, sería una experiencia mucho más enriquecedora que trataré de implementar en mi método habitual. Otro apartado muy importante ha sido la reflexión acerca de los contenidos impartidos. La esquematización de los contenidos y sus interrelaciones con las que hemos trabajado para el ciclo de mejora me ha ayudado mucho a comprender qué es realmente lo esencial y qué es información superflua o de apoyo. Estos mapas de contenidos me han servido además para identificar las preguntas clave que van dando lugar a los distintos tipos de contenidos, algo que me ha servido en el desarrollo de la clase. Hasta ahora nunca antes había creado un mapa de contenidos, pero creo que es algo a lo que realmente merece la pena dedicar un tiempo para mejorar la calidad de la docencia.

Ciclos de Mejora en el Aula (2020). Experiencias de Innovación Docente de la US Esta obra se distribuye con la licencia Creative Commons 
Respecto a la metodología empleada en las clases, creo que es fundamental la implicación activa de los alumnos. Ya sea mediante trabajos fuera del aula o mediante debates individuales o por grupos, los alumnos deben tener el protagonismo en la clase, y eso es algo a lo que quiero dar la mayor importancia en el futuro. Para ello, el tener pensado de antemano cómo espero que se desarrolle la clase y detallado en una secuencia de actividades es algo muy útil para no dejarse llevar por una actividad en concreto y que todo lo planificado inicialmente quede mucho más compensado. Me ha servido mucho el usar un caso práctico como hilo conductor de un bloque de contenidos, y creo que el pararse a pensar un buen ejemplo que sea capaz de llamar la atención de los estudiantes y motivarles a trabajar en la asignatura puede resultar determinante para alcanzar unos buenos resultados en el proceso de aprendizaje.

Por último, creo especialmente relevante el hecho de emplear múltiples aproximaciones para evaluar el aprendizaje y tener así una calificación final más ajustada a los conocimientos adquiridos por los estudiantes. A partir de diversos trabajos entregables de forma regular se puede determinar de una manera mucho más precisa el grado de conocimiento de cada alumno. Este tipo de trabajo continuo creo que es mucho más efectivo a la hora de evaluar y creo que se quedará en mi metodología habitual.

\section{Principios didácticos que deben permanecer}

En primer lugar, lo que me ha quedado claro es la necesidad imperiosa de implicar más al alumnado en el proceso de enseñanza. He descubierto numerosas actividades de contraste con las que se pueden ir desarrollando los contenidos, tanto de forma individual como en pequeños grupos, de forma que los estudiantes puedan ir formando 
sus propias ideas y adquieran un aprendizaje más maduro. El hecho de tratar de hablar menos en clase y dar más protagonismo a los alumnos es uno de mis propósitos más firmes después de la implementación del CIMA aquí expuesto. Segundo, creo que es muy necesario hacer una evaluación crítica de los contenidos de la materia, de forma que se establezca una clara jerarquía y priorización entre los distintos de contenidos (actitudinales, estructurales, secundarios o procedimentales). De este modo, se podrá dar la importancia adecuada a cada tipo de contenido y facilitará la interconexión entre ellos. Por último, los cuestionarios iniciales me han parecido una forma muy clara de conocer los modelos mentales de los estudiantes y los principales obstáculos de aprendizaje, para poder adaptar los contenidos de forma que se salven esos obstáculos. Reconozco que el hecho de crear las escaleras de aprendizaje es algo que consume bastante tiempo, pero aporta una información muy valiosa.

Ciclos de Mejora en el Aula (2020). Experiencias de Innovación Docente de la US Esta obra se distribuye con la licencia Creative Commons 
Palabras clave: Ciencia y Tecnología del Medio Ambiente, Grado en Ingeniería Agrícola, Docencia universitaria, Experimentación docente universitaria, Ecología.

Keywords: Environmental Science and Technology, Bachelor in Agricultural Engineering, University teaching, University teaching experimentation, Ecology.

\section{Referencias bibliográficas}

Cambrollé Silva, J. (2018). Aplicación de un Ciclo de Mejora Docente en Ciencia y Tecnología del Medio ambiente. Jornadas de Formación e Innovación Docente del Profesorado, 1, 1272-1289.

Finkel, D. (2008). Dar clase con la boca cerrada. Valencia: Universitat de València.

García, E., Porlán, R., \& Navarro, E. (2017). Los fines y los contenidos. Enseñanza universitaria. Cómo mejorarla, 55-72.

Mancilla Leytón, J. M. (2018). Análisis de la implementación de un ciclo de mejora docente en la asignatura Ciencia y Tecnología del Medio Ambiente. Jornadas de Formación e Innovación Docente del Profesorado, 1, 1376-1393.

Porlán, R. (Coord.) (2017). Enseñanza Universitaria. Cómo mejorarla. Madrid: Ediciones Morata.

Rivero, A., \& Porlán, R. (2017). La evaluación en la enseñanza universitaria. Enseñanza universitaria. Cómo mejorarla, 73-91.

Ciclos de Mejora en el Aula (2020). Experiencias de Innovación Docente de la US Esta obra se distribuye con la licencia Creative Commons 